\title{
TECNOLOGIAS MÓVEIS: uma mais valia em contextos educacionais?
}

\author{
Katja Göttsche*
}

\section{Resumo}

Após uma sucinta introdução à temática, apresentam-se algumas perspectivas sobre a aprendizagem com tecnologias móveis. De seguida questiona-se o potencial da aplicação das tecnologias móveis em contextos educacionais, reflectindo-se nomeadamente sobre o desenvolvimento e desenho de actividades educacionais com tecnologias móveis, sobre a avaliação deste género de actividades e sobre o modo de integração na escola das tecnologias móveis. As reflexões finais abordam questões de ordem ética relacionadas com a aprendizagem com tecnologias móveis em contextos educacionais.

Palavras-chave: Aprendizagem móvel. Escola. Actividades educacionais. Questões éticas.

\section{HUMAN BEINGS/TECHNOLOGIES COUPLING: breaking with the purification of modernity}

\begin{abstract}
After a short introduction to the topic, some perspectives on learning with mobile technologies are presented followed by some questions concerning the potential of the application of mobile technologies in educational contexts, with a special focus on the development and design of learning activities for mobile devices, on the evaluation of this kind of learning activities and on the way mobile technologies should be integrated into schools. The final reflections concern ethical issues related to learning with mobile technologies in educational contexts.
\end{abstract}

Keywords: Mobile learning. Mobile technologies in the classroom. Learning activities.

\section{Introdução}

Perante o boom que a aprendizagem móvel conheceu nos últimos anos e em face das inúmeras (e por vezes muito diversas) definições de aprendizagem móvel (m-learning) existentes, pareceu necessário a autores como Niall Winter reconceptualizar aquele conceito de modo a poder compreender a sua natureza específica (WINTER, 2007, p. 7). Naismith et al. (2004, p. 9) constatam igualmente um défice teórico recorrente no que se refere à

\footnotetext{
* Doutoranda em Educação e Professora da Universidade Aberta de Portugal. E-mail: kgottsch@uab.pt
} 
utilização das tecnologias móveis em contextos educacionais. Por um lado, esta multiplicidade de perspectivas é sem dúvida positiva e enriquecedora, mas por outro lado, a verdade é que a diversidade daí decorrente dificulta as tentativas de desenvolver uma teoria da aprendizagem móvel (WINTER, 2007, p. 7).

O’Malley et al. (2005 apud WISHART, 2007, p. 62) propõem que as teorias da aprendizagem móvel sejam verificadas e testadas a partir de uma série de questões: a teoria da aprendizagem com tecnologias móveis abrange contextos educacionais formais e informais? O contexto educacional dinâmico é analisado? A aprendizagem é concebida como uma actividade construtiva e social?

Em definições anteriores de aprendizagem móvel, é sobretudo à perspectiva tecnocêntrica que cabe o papel mais importante, i.e., a aprendizagem móvel é entendida como (mais) uma oferta de ensino na qual são utilizadas, preferencial ou mesmo exclusivamente, tecnologias móveis, como Palmtops ou outros aparelhos semelhantes (TRAXLER, 2005 apud SPECHT, 2011, p. 2).

A perspectiva tecnocêntrica deu posteriormente lugar a uma perspectiva centrada no aprendente, constituindo este, e em especial a sua mobilidade, o critério central de definições, como por exemplo aquela que O’Malley propõe de aprendizagem móvel: “Any sort of learning that happens when the learner is not at a fixed, predetermined location, or learning that happens when the learner takes advantage of learning opportunities offered by mobile technologies"” (O’MALLEY et al., 2003 apud WINTER, 2007, p. 8; SPECHT, 2011, p. 2).

Paralelamente existem perspectivas que veêm este tipo de aprendizagem como uma simples extensão do e-learning, perspectivas essas que não reconhecem portanto o carácter específico da aprendizagem móvel (WINTER, 2007, p. 7).

Autores como Sharples (2005) e Taylor et al. (2006) procuram desenvolver uma teoria da aprendizagem móvel com base nas noções de Activity Theory (ENGELSTRÖM, 1999) e Conversational Framework (LAURILLARD, 2002). A aprendizagem móvel é então aí entendida como comunicação em contexto (WINTER, 2007, p. 8).

Enquanto que há dez anos a questão de saber como os conteúdos podiam ser disponibilizados em aparelhos móveis constituía uma questão técnica central, actualmente, a integração e harmonização de tecnologias móveis nos modelos correntes de apoio à aprendizagem tende a assumir um papel de primeiro plano (SPECHT, 2011, p. 2).

\footnotetext{
1 "Qualquer tipo de aprendizagem que acontece quando o aluno não está em um local fixo, pré-determinado, ou a aprendizagem que acontece quando o aluno aproveita oportunidades de aprendizagem oferecidas pelas tecnologias móveis." Tradução das organizadoras.
} 
Consequentemente, na investigação mais recente, conceitos como 'contextualização', 'personalização', 'interacção multimédia', ‘awareness' e 'reflexão’ são considerados componentes centrais de uma aprendizagem móvel (SPECHT, 2011, p. 2).

Um papel importante é atribuído também à natureza transformativa das tecnologias móveis, quer dizer, à questão de saber até que ponto as tecnologias móveis possibilitam ao aprendente obter resultados a que ele não chegaria sem a utilização dessas mesmas tecnologias (WINTER, 2007, p. 8).

Se a aprendizagem móvel for entendida como uma rede de aprendizagem distribuída (distributed learning network), então a aprendizagem móvel é uma parte de um todo maior, onde as tecnologias de aprendizagem, as actividades e os contextos educacionais, bem como as pessoas estão distribuídas no tempo e no espaço. Neste âmbito levantam-se importantes questões relativas à socialização, representação e personalização (WINTER, 2007, p. 9), em torno das quais se desenvolve este texto.

\section{Tecnologias móveis em contextos educacionais}

Qual o potencial da aplicação das tecnologias móveis em contextos educacionais? 0 que torna as tecnologias móveis tão interessantes para tantos aprendentes?

Em Klopfer et al. (2002) são identificadas cinco características das tecnologias móveis que possuem um potencial único para os objectivos educacionais: portabilidade, interactividade, sensitividade de contexto, conectividade e individualidade (KLOPFER et al., 2002 apud NAISMITH, 2004, p. 9).

Segundo Klopfer, as tecnologias móveis possibilitam a aprendizagem em contextos muito variados, o intercâmbio de dados, o trabalho conjunto com outros aprendentes, a acumulação de dados e a criação de redes comuns. A natureza transformativa das tecnologias móveis, quando aplicada de uma forma consequente e determinada, pode proporcionar ao aprendente experiências e processos de aprendizagem completamente novos, que lhe seriam desconhecidos antes.

Sobretudo os mais jovens acham as tecnologias móveis especialmente apelativas e adequadas às suas necessidades e usos individuais, e mostram-se assim também mais motivados para aprender com este género de tecnologias. Como se pode explicar esta motivação? Jones refere como possível causa o facto de o aprendente ter um controle sobre o processo e as metas da aprendizagem, a apropriação das tecnologias móveis, o divertimento 
que proporcionam, as possibilidades de comunicação que oferecem, a possibilidade de aprendizagem em contextos muito diversos, mas também outros factores de identificação como “coolness”. A apropriação das tecnologias móveis parece ter um papel particularmente importante na motivação do aprendente (JONES et al., 2006 apud JONES, 2007, p. 18). Além disso, as tecnologias móveis possibilitam acesso imediato aos dados, facto que seguramente contribui também para aumentar a motivação.

Os factores afectivos - tanto positivos, como negativos - desempenham reconhecidamente um papel importante no grau de empenhamento, assim como no grau de satisfação do aprendente.

Naismith et al. (2004) proporcionam uma perspectiva ampla sobre diversas actividades de aprendizagem com tecnologias móveis na sala de aula e fora dela, subdividindo as diversas formas de aprendizagem com tecnologias móveis segundo diferentes concepções de aprendizagem e teorias pedagógicas em abordagens behaviorista, construtivista, situada, colaborativa e aprendizagem informal. Proporcionam ainda exemplos da utilização de tecnologias móveis para coordenação dos aprendentes e dos recursos. Os mesmo autores adoptam uma classificação das tecnologias móveis que se define em função dos eixos portátil versus estático e pessoal versus partilhado (NAISMITH et al., 2004, pp. 7-8). Dentro desta classificação são consideradas como tecnologias portáteis e pessoais os telemóveis, os PDAs, tablet PCs, computadores portáteis e ainda as consolas de jogos portáteis.

Como deveriam ser desenhadas as actividades de aprendizagem com tecnologias móveis de modo a produzirem uma efectiva melhoria do processo de aprendizagem e dos seus resultados?

Quanto ao design de actividades de aprendizagem para tecnologias móveis, como suporte para práticas educacionais, Milrad (2007, p. 29) salienta que se torna fundamental conjugar as teorias de aprendizagem, o desenho e o uso educacional na adaptação das tecnologias móveis a contextos educacionais.

Hoppe (2007, p. 32) salienta que, antes do mais, as tecnologias móveis devem ser sempre utilizadas como suporte para objectivos educacionais bem fundamentados e previamente definidos.

Para desenhar práticas educacionais inovadoras torna-se por conseguinte necessário adoptar uma perspectiva integrada da aprendizagem apoiada em tecnologias, perspectiva essa onde a pedagogia e a teoria de aprendizagem - mais, em todo o caso, do que a tecnologia móvel - devem ser consideradas as verdadeiras forças motrizes de todo o processo 
(MILRAD, 2007, p. 31).

No que se refere ao design, deve igualmente saber-se como determinadas tecnologias têm de facto uma natureza transformativa, isto é: será importante saber até que ponto a utilização de tecnologias móveis permite ao aprendente alcançar resultados que não seriam passíveis de ser obtidos sem estas tecnologias (WINTER, 2007, p. 8).

Uma questão importante que se coloca ao longo do processo de design de actividades educacionais com tecnologias móveis é portanto a de saber se a ideia educativa original está adequadamente harmonizada, isto é, se a ideia educativa é suportada pela tecnologia móvel adoptada (HOPPE, 2007, p. 35).

Como refere ainda o mesmo autor (HOPPE, 2007, p. 31), as necessidades específicas da aprendizagem com tecnologias móveis devem ser perspectivadas em contextos educacionais alargados. Nestes cenários educacionais alargados, as tecnologias móveis inter operam tanto com tecnologias ubíquas como com as infraestruturas das redes e do servidor.

Neste contexto é preciso identificar as potencialidades específicas (affordances) e as limitações inerentes às diversas tecnologias móveis para definir e distribuir adequadamente a funcionalidade das respectivas aplicações móveis em função dos objectivos educacionais, com o intuito de melhorar efectivamente o processo de aprendizagem.

A título de exemplo: pequenas tecnologias móveis como smartphones, podem não ser a ferramenta mais adequada para processar informações visuais ou gráficas; no entanto, podem ser adequados para actividades com base em áudios, na aprendizagem de línguas. (HOPPE, 2007, p. 32; MOURA, 2010).

Quais as implicações da aprendizagem com tecnologias móveis para a avaliação (da aprendizagem)? Quais os aspectos a considerar?

Embora existam métodos testados e comprovados para a avaliação de diversas aplicações técnicas, não existe até agora um enquadramento global, completo e exaustivo para uma avaliação formativa alargada em um ambiente de aprendizagem móvel, dado tratar-se de um fenómeno ainda relativamente recente (TAYLOR, 2007, p. 29). Além disso, a aprendizagem móvel acontece frequentemente fora de contextos educacionais formais, ou seja, em contextos informais de aprendizagem, onde a questão da avaliação não se coloca, pelo menos do mesmo modo que se coloca em contextos formais.

Tendo em conta a progressiva personalização dos ambientes de aprendizagem no contexto das tecnologias móveis, torna-se difícil, por um lado, prever possíveis percursos de 
aprendizagem, do mesmo modo que se revela difícil, por outro lado, compreender esses percursos e processos, ou mesmo identificar as suas fontes. Assim, em trabalhos de grupo, por exemplo, os participantes com ambientes de aprendizagem individuais podem seleccionar e fazer uso de tecnologias diferentes, chegando eventualmente a resultados diversos por caminhos também eles diferentes (TAYLOR, 2007, p. 28).

Afigura-se assim necessário desenvolver métodos que possam ser adequados aos desafios específicos da aprendizagem móvel, métodos esses a partir dos quais as actividades de aprendizagem em ambientes educacionais personalizados possam ser correctamente identificadas, descritas, interpretadas e avaliadas.

De acordo com Taylor (2007, p. 28) a avaliação devia fazer parte do processo de design, logo desde o seu início, e podia ter a forma de uma autoavaliação do aprendente através de mecanismos de feedback automatizado (aliás, neste contexto, Taylor refere-se a uma série de possíveis métodos de avaliação).

Além disso, a noção de learning-outcomes devia ser repensada e reformulada no contexto de aprendizagem móvel, e desse modo ser adaptada ao contexto educacional diferenciado deste género de aprendizagem.

A par disso é importante perceber ainda como as actividades educacionais estruturadas se fundem com as actividades mais sociais ou informais na aprendizagem com tecnologias móveis (TAYLOR, 2007, p. 28).

\section{Como integrar a aprendizagem com tecnologias móveis no contexto escolar? E quais as questões éticas que se levantam?}

De acordo com a visão de Naismith (2004, p. 36), num futuro próximo as tecnologias móveis hão-de tornar-se cada vez mais ubíquas, hão-de estar cada vez mais integradas e em rede, proporcionando funcionalidades contextuais que poderão transformar qualquer actividade do dia a dia, na medida em que tornam possível capturar informações detalhadas relativamente ao tempo, à localização, às pessoas à volta do utilizador e às condições atmosféricas, entre outras. Futuramente as oportunidades para a colaboração distribuída e o trabalho móvel em equipa serão ainda mais facilitadas pelas tecnologias móveis.

Estas mesmas tecnologias, como afirma ainda a mesma autora, terão um impacto enorme na forma como se vai aprender. A aprendizagem terá lugar cada vez mais fora da sala de aula e cada vez mais no ambiente personalizado do aprendente, seja este real ou virtual, e muitas vezes em contextos informais de aprendizagem. 
Já hoje em dia, fora do contexto escolar, os jovens fazem uso das tecnologias móveis através da partilha de media e das redes sociais, desenvolvendo capacidades importantes para a sua futura vida profissional, tais como knowledge working, media production e o trabalho em equipa.

Porém, actualmente, a maior parte das escolas dispensa a utilização das tecnologias móveis para fins educacionais e proíbe mesmo o uso de telemóveis e computadores pessoais na escola, que são sobretudo vistos como factor disruptivo e de distracção. A maior parte das escolas ainda não se adaptou a um contexto social diferente, com novas formas de comunicação, de ensino e de trabalho, um contexto com redes sociais e tecnologias móveis.

É notória uma tensão sistémica entre o uso corrente e quotidiano das TICs pelas crianças e pelos jovens, em especial das tecnologias móveis e a aula tradicional (SHARPLES, 2007, p. 23). O encontro de dois contextos educacionais, o formal e o informal, pode eventualmente transformar-se num confronto entre a sala de aula, com um ensino ainda frequentemente centrado no docente, por um lado, e uma cultura teenager, caracterizada por teamworking, sharing, social networking e aprendizagem colaborativa, por outro.

Como podem as escolas integrar a aprendizagem com tecnologias móveis no currículo e desse modo aproveitar as capacidades que as crianças e jovens adquiriram com a sua utilização?

Em primeiro lugar, torna-se importante entender melhor todo o leque de experiências de aprendizagem que as crianças e jovens fazem no seu uso informal das tecnologias móveis fora do contexto escolar e, em segundo lugar, é preciso reconhecer e valorizar estas aprendizagens em contextos informais e as skills aí adquiridas. Os estudos realizados nesta área revelam que muitos jovens estão habituados a trabalhar em comunidades de prática ou comunidades de aprendizagem, onde assumem os papéis tanto de professor como de aprendente (induzindo ainda outras pessoas às suas actividades), enquanto outros jovens aprendem activamente um leque de skills e competências que fazem parte da cultura do seu peer group ou mediatizados por tecnologias digitais. Estes hábitos de aprendizagem devem ser reconhecidos e valorizados para serem desenvolvidos de forma sistemática nas escolas e no curriculo com o intuito de desenvolver e melhorar o sistema educacional.

Para além disso torna-se importante também perceber como os aprendentes transferem skills, competências e saberes adquiridos com tecnologias móveis em contextos de aprendizagem informal para contextos educacionais de aprendizagem formal (SEFTONGREEN, 2004, p.31). 
Na base de uma integração bem sucedida da aprendizagem com tecnologias móveis na escola está a criação de uma nova cultura TIC a um nível institucional, quer dizer, a utilização de tecnologias móveis deve ser acompanhada e sustentada por um modelo pedagógico forte. Entre outras coisas essa integração exige a identificação de objectivos educativos claros, uma formação aprofundada dos professores na utilização dos TICs, e a criação de uma infraestrutura técnica sólida (NAISMITH et al., 2004, p. 33-35). Em situação ideal a escola devia disponibilizar as tecnologias móveis (pelo menos às crianças mais desfavorecidas) e assim contribuir para ultrapassar o chamado digital gap.

As escolas deveriam integrar a aprendizagem com tecnologias móveis no currículo com base num modelo pedagógico bem definido e produtivo, aproveitando assim as capacidades que os aprendentes demonstram ter, e fazendo uso delas para fins educacionais e de aprendizagem. As crianças e jovens, por seu turno, deveriam aprender a adaptar as suas práticas de networking ao contexto escolar, apoiadas por ferramentas e tecnologias móveis que promovam o teamworking e a aprendizagem colaborativa (SHARPLES, 2007, p. 25).

A par destes aspectos as crianças e jovens aprenderiam a ser capazes de lidar de uma forma construtiva e responsável com as tecnologias móveis, uma questão que se afigura hoje absolutamente central numa sociedade onde as TICs estão cada vez mais presentes.

Uma questão que se afigura como central no âmbito da investigação das TICs é, se e em que medida, a introdução das tecnologias digitais em muitos aspectos do nosso dia a dia, formando parte do nosso mundo do trabalho, dos tempos livres, e de experiências educacionais pode levar à exclusão das pessoas que não podem ou não querem obter e utilizar estas tecnologias. $\mathrm{O}$ factor que determina a propriedade de tecnologias digitais continua a ser a classe social, dispondo as famílias mais abastadas de significativamente mais tecnologias digitais (SEFTON-GREEN, 2004, p. 19). Enquanto o telemóvel parece ser uma tecnologia relativamente “democrática”, o acesso à internet continua a ser mais restrito (SEFTONGREEN, 2004, p. 19).

Perante esta situação, (como) pode a instituição escola contribuir para uma literacia digital, nomeadamente dos alunos socialmente desfavorecidos? Pode a instituição escola contribuir para ultrapassar o digital gap promovendo a utilização das tecnologias móveis em contextos educacionais?

Naismith refere que para as escolas torna-se geralmente menos caro equipar cada estudante com uma tecnologia móvel (como um handheld computer) do que adquirir computadores fixos ou computadores portáteis. A autora mantém que a tecnologia móvel pode de facto ser uma medida para encarar a questão do "digital divide”, sendo as tecnologias 
móveis a forma mais económica para equipar os alunos com uma ferramenta digital, que pode ser levada para casa e através da qual os alunos podem aderir à internet (PERRY, 2003 in NAISMITH, 2004, p. 34). Além disso, o carácter pessoal e colaborativo das tecnologias móveis pode ainda encorajar a participação e a motivação de alunos desinteressados ou de estudantes em risco (TRAXLER, 2004 in NAISMITH, 2004, p.34).

Várias tecnologias móveis possibilitam o registo digital do seu utilizador e do seu meio e o registo da comunicação ao nível da sala de aula (virtual), o que levanta uma série de questões éticas significativas relativamente ao registo, à storage armazenamento seguro dessas informações, como o intuito de evitar o acesso a e o mau uso dessas informações por terceiros (NAISMITH, 2004, p.33). As escolas e os desenhadores das tecnologias móveis terão que dar resposta à questão da protecção da privacidade e segurança do utilizador.

Será necessário além do mais esclarecer quais as fronteiras da escola e quais as zonas em que não se afigura legítima a intervenção da escola na infância. Será por exemplo legítimo controlar e monitorizar os alunos por meio do software das tecnologias móveis em actividades educacionais fora do espaço da aula (SHARPLES, 2004, p. 4; 25)?

As tecnologias móveis possibilitam o registo digital das nossas vidas e das de outros. Como pode ser garantido o direito à privacidade, à integridade e à segurança na época das tecnologias móveis? E quando é que esses direitos são feridos? Quem é detentor por exemplo dos direitos sobre os registos das nossas vivências (NAISMITH, 2004, p. 33)?

As perspectivas sociais do público e do privado transformaram-se sob influência dos novos média digitais e, muito em particular, sob influência das tecnologias móveis. Há zonas ambíguas e indefinidas. Neste contexto, a tarefa das escolas, no quadro de uma pedagogia dos média, consiste em fazer com que as crianças e jovens aprendam a lidar com as tecnologias móveis de uma forma responsável, madura e ética, e que na aprendizagem com tecnologias móveis em contextos educacionais fiquem garantidos os direitos à privacidade, integridade e segurança de todos os intervenientes no processo de aprendizagem, alunos bem como professores (NAISMITH, 2004, p. 33-35).

Para além das questões éticas que foram levantadas, existem argumentos legítimos contra a útilização das tecnologias móveis nas escolas, referidos por exemplo pelo Consortium for School Networking (2012, p.5). Como argumentos contra a utilização das tecnologias móveis nas escolas, os autores desse estudo referem, entre outros, as práticas de sexting, isto é, a divulgação de mensagens ou imagens sexualmente explícitas por telemóveis e as frequentes práticas de cyberbulling e harassment nos media sociais. Salientam ainda a frequente falta de capacidade de juizo crítico das crianças e jovens quanto às informações que 
podem ou não partilhar online com outros.

\section{A concluir}

Procurámos ao longo deste texto discutir um conjunto de aspectos que julgamos necessitam ser equacionados perante a realidade que o uso das tecnologias móveis vem, cada vez, produzindo. Para além do seu potencial, interessou-nos também considerar aspectos problemáticos da sua utilização, visando uma reflexão suficientemente ampla da questão.

Tal como os autores do Consortium for School Networking (2012, p.6-7) consideramos que a proibição das tecnologias móveis nas escolas não é a resposta adequada. Os mesmos autores recomendam que as condições de utilização deveriam ser revistas e eventualmente alteradas, devendo adoptar-se uma política de utilização responsável nas escolas. Deveria ser aproveitada a oportunidade de tornar os alunos competentes na utilização das tecnologias móveis, salientando-se ainda a importância disso para o seu futuro desenvolvimento profissional.

Em suma, conforme afirma Naismith (2004, p.36), sejam elas bem-vindas ou não, a verdade é que as tecnologias móveis hão-de entrar nas salas de aula nos bolsos das crianças e jovens. O grande desafio que se coloca para as escolas e para os educadores é conseguir incluir estas tecnologias de forma produtiva nas suas práticas educativas.

\section{Referências}

ENGELSTRÖM,Y. Perspectives on Activity Theory (Learning in Doing: Social, Cognitive \& Computational Perspectives). Cambridge: Cambridge University Press, 1999.

HOPPE, U. How can we integrate mobile devices with broader educational scenarios? In: SHARPLES, M. (ed.). Big Issues in Mobile learning - Report of a workshop by the Kaleidoscope Network of Excellence Mobile Learning Initiative. Learning Sciences Research Institute, University of Nottingham, 2007, p. 32-35. Disponível em. http://mlearning.noekaleidoscope.org/repository/BigIssues.pdf. Acesso em: 25 mar. 2012.

JONES, A / ISSROFF, K. / SCANLON, E. / CLOUGH, G. / McANDREW, P. Using mobile devices for learning in Informal Settings: Is it Motivating? (Paper to be presented at IADIS International Conference Mobile Learning. July 14-16 Dublin, 2006).

JONES, A. / ISSROFF, K. / SCANLON, E. Affective factors in learning with mobile devices. In: SHARPLES, M. (ed.) Big Issues in Mobile learning - Report of a workshop by the Kaleidoscope Network of Excellence Mobile Learning Initiative. Learning Sciences Research Institute, University of Nottingham, 2007, p. 17-22. Disponível em: http://mlearning.noekaleidoscope.org/repository/BigIssues.pdf. Acesso em: 25 mar. 2012. 
KLOPFER, E. / SQUIRE, K. / JENKINS, H. Environmental Detectives: PDAs as a window into a virtual simulated World. Proceedings of IEEE International Workshop on Wireless and Mobile Technologies in Education. Vaxjo, Sweden: IEEE Computer Society, 2002 (pp. 95-98).

LAURILLARD, D. Rethinking University Teaching: a conversational framework for the effective use of learning tecnologies. London: Routledge, Falmer 2002.

MAKING PROGRESS: Rethinking State and School Districts Policies Concerning Mobile Technologies and Social Media. Disponível em: http://www.cosn.org/Initiatives/ParticipatoryLearning/Home/tabid/7112/Default.aspx. Acesso em: 7 out. 2012.

MILRAD, M. How should learning activities using mobile tecnologies be designed to support innovative educational practices? In: SHARPLES, M. (ed.). Big Issues in Mobile learning - Report of a workshop by the Kaleidoscope Network of Excellence Mobile Learning Initiative. Learning Sciences Research Institute, University of Nottingham, 2007, p. 29-31.

Disponível em: http://mlearning.noe-kaleidoscope.org/repository/BigIssues.pdf. Acesso em: 25 mar. 2012.

MOURA, A. Geração Móvel: um ambiente de aprendizagem suportado por tecnologias móveis para a “Geração Polegar”, 2010. Disponível em: http://adelinamouravitae.com.sapo.pt/gpolegar.pdf. Acesso em: 25 mar. 2012.

NAISMITH, L./ LONSDALE, P. / VAVOULA, G. / SHARPELS, M. Literature Review in mobile Technologies and Learning, Futurelab Series, Report 11, 2004 Disponível em: http://www.futurelab.org.uk/resources/mobile-technologies-and-learning-literature-review. Acesso em: 25 mar.2012.

O’MALLEY et al. Pedagogical methodologies and paradigms: Guidelines for learning/teaching/tutoring in a mobile environment. MOBIlearn Report UoN, UoB,OU/WP4/1.2., 2005.

PERRY, D. Hand-held Computers (PDAs) in Schools. Coventry, UK: Becta (for DfES), 2003. Disponível em: http://www.becta.org.uk/research/research.cfm?section=1\&id=541. Acesso em: 25 mar.2012.

SHARPLES, M. (ed.) Big Issues in Mobile learning - Report of a workshop by the Kaleidoscope Network of Excellence Mobile Learning Initiative. Learning Sciences Research Institute, University of Nottingham, 2007. Disponível em: http://mlearning.noe-kaleidoscope.org/repository/BigIssues.pdf. Acesso em: 25 mar. 2012.

SPECHT, M. Mobiles und Ubiquitäres Lernen. Technologien und didaktische Aspekte. In: EBNER, M. / SCHÖN, S. (Eds.), Lehrbuch für Lernen und Lehren mit Technologien (L3T), 2011. Disponível em: http://13t.tugraz.at/index.php/LehrbuchEbner10/article/view/74/36. Acesso em: 25 mar. 2012.

TAYLOR, J. Evaluating Mobile Learning: What are appropriate methods for evaluating learning in mobile environments? In: SHARPLES, M. (ed.) Big Issues in Mobile learning - Report of a workshop by the Kaleidoscope Network of Excellence Mobile Learning Initiative. Learning Sciences Research Institute, University of Nottingham, 2007. p. 26-28. Disponível em: http://mlearning.noekaleidoscope.org/repository/BigIssues.pdf. Acesso em: 25 mar. 2012.

TRAXLER, J. Mobile Learning - Content and Delivery (presentation). Telford, UK: Learning Lab, 2004. 
TRAXLOR, J. Mobile Learning - it's here but what is it? Interactions 9, 1. Warwick, University of Warwick, 2005.

WINTER, N. What is mobile learning? In: SHARPLES, M. (ed.) Big Issues in Mobile learning Report of a workshop by the Kaleidoscope Network of Excellence Mobile Learning Initiative. Learning Sciences Research Institute, University of Nottingham, 2007, p. 7-11. Disponível em:

http://mlearning.noe-kaleidoscope.org/repository/BigIssues.pdf. Acesso em: 25 mar.2012.

WISHART, J. The Seven Cs - no eight - no nine „C“s of m-learning. In: ARNEDILLO-SÁNCHEZ, I. / SHARPELS, M. / VOVOULA, G. (Eds.) Beyond Mobile Learning Workshop, The CSCL Alpine Rendez-Vous, kaleidoscope Mobile Learning SIG, Trinity College Dublin Press, 2007, p. 58-63. Disponivel em: http://mlearning.noe-kaleidoscope.org. Acesso em: 25 mar.2012. 